\title{
Soñando el futuro. Mujeres estudiantes y profesionales en Cádiz en torno a $1900^{1}$
}

Dreaming of the future. Female students and professionals in Cádiz around 1900

Consuelo Flecha García

Universidad de Sevilla

cflecha@us.es

\section{Resumen}

En estas páginas se hace un recorrido por el itinerario de estudios y profesional de algunas mujeres que, bien nacieron, bien estudiaron, o bien trabajaron en Cádiz. Médicas, Farmacéuticas, Maestras y una Inspectora; transitaron muy solitariamente por la Facultad de Medicina gaditana, que estrenaron el ejercicio de determinadas profesiones en la ciudad, y que despertaron curiosidad si nos fijamos en su presencia en la prensa. Traer su memoria al presente ha sido el objetivo del trabajo. Se han utilizado fuentes bibliográficas, hemerográficas y archivísticas siguiendo los criterios de la investigación histórica.

Palabras clave: Maestras. Universitarias. Profesionales. Cádiz. XIX. XX

\begin{abstract}
These pages take a tour of the study and professional itinerary of some women who were either born, studied, or worked in Cádiz. Doctors, Pharmacists, Teachers and an Inspector. That they walked very lonely through the Cadiz Faculty of Medicine, that they released the exercise of certain professions in the city, and that aroused curiosity, if we look at their presence in the press. Bringing his memory to the present has been the objective of the work. Bibliographic, newspaper and archival sources have been used following the criteria of historical research.
\end{abstract}

Keywords: Teachers. University. Professionals. Cadiz. XIX. XX

${ }^{1}$ Recibido: 2021 Evaluado: 4/02/2021 Aceptado: 19/03/2021 


\section{Introducción}

Una mujer nacida en Cádiz que alcanzó notable relevancia en su amplia y activa vida profesional, María Encarnación de la Rigada Ramón (Cádiz, 1863-Madrid, 1930), profesora de la Escuela Normal Central de Maestras, escribe en el periódico sobre temas de enseñanza del que era directora y propietaria, el orgullo de haber tenido, a la primera bibliotecaria que ganaba una plaza por oposición, como alumna. Se refería a la licenciada en Filosofía y Letras desde 1912 Ángela García Rives (Madrid, 1891-1968?), evocando "con gran satisfacción que ha sido una de las más aventajadas discípulas de la directora de Gaceta de Instrucción Pública y Bellas Artes en aquella época en que la distinguida bibliotecaria de hoy, cursó con lucimiento, sus estudios para maestra en la Normal de Madrid” (Rigada, 1913, p. 519).

Ambas representan muy bien a dos generaciones de mujeres que ejercieron en España campos de actividad cuyo desempeño requería una formación académica específica. En el primer caso, María Encarnación, el grado más alto impartido en la Escuela Normal Central de Maestras desde 1882, que en las Escuelas Normales de Maestros había sido creado por la Ley Moyano de 1857 como requisito para incorporarse después al profesorado de las mismas; el Grado Normal. Y en el segundo caso, la licenciatura universitaria, para optar a plazas de Bibliotecas, Museos y Archivos, o del profesorado de Institutos de Segunda Enseñanza y de la Universidad; puestos profesionales a los que sólo pudieron incorporarse las mujeres a partir de la R.O. de 11 de junio de 1910. Sí llevaban tres décadas ejerciendo la medicina y, algo menos, como farmacéuticas, pero el gran despliegue y crecimiento en actividades con necesidad de cualificación superior se iba a producir durante el primer tercio del siglo $\mathrm{XX}$.

En las jóvenes de muchas familias de clase media, decididas a invertir recursos en los estudios, no solo de sus hijos, también de sus hijas, ese proceder desencadenó un viraje decisivo en el destino adjudicado a las mujeres. Y ellas encontraron así un camino más expedito para elegir, bien una vida familiar propia, de esposas y de madres, o bien una soltería no sobrevenida por circunstancias ajenas a su deseo, sino adoptada por voluntad propia. Porque los estudios conducían al ejercicio de una profesión, trabajo remunerado que, en cualquiera de las dos opciones, les daba mayor autonomía y el poder valerse sin el concurso de otras personas.

Son numerosas las publicaciones que tratan sobre el acceso a distintos estudios reglados, a profesiones y presencias en compromisos de carácter público, durante las últimas décadas del siglo XIX y primeras del XX. Curiosidad investigadora que ha proporcionado datos concretos e información sistemática sobre cómo se produjo este hecho en distintos países. Centrándonos en España, la obra que desveló una realidad educativa y profesional muy olvidada fue la de Rosa María Capel sobre el trabajo y la educación de las mujeres en el primer tercio del siglo XX (Capel, 1982). Libro lleno de resultados y de fuentes que despertaron el deseo de ampliar, profundizar, conocer más, de lo mucho que aquellas páginas brindaban. La producción ha sido abundante, valiosa, estimulante para una población femenina que ignoraba una parte sustancial de su historia y que había sido convencida de la ignorancia secular de las mujeres, así como de su incapacidad para

$\mathrm{N}^{\circ}$ 9, 2021. Página | 18 
funciones externas a las de naturaleza familiar; en consecuencia, dirigidas unidireccionalmente a una actividad previsible, sin aristas en su destino.

Hoy disponemos de biografías de maestras, inspectoras de primera enseñanza, profesoras de Escuelas Normales e Institutos de Segunda Enseñanza, bibliotecarias, médicas, farmacéuticas, abogadas; de las primeras profesionales en cada uno de estos sectores. Conocemos como se inició la formación de las maestras en el siglo XIX, el acceso de alumnas a Institutos de Segunda Enseñanza, a carreras universitarias, las primeras ejerciendo la medicina, al frente de oficinas de Farmacia, como opositoras al profesorado de los diferentes niveles de enseñanza y a otros organismos de la administración pública, dedicando un tiempo de su vida a la investigación o ejerciendo -son menos en aquel tiempo- la abogacía.

Cuando en 1936 se interrumpe trágicamente la cotidianidad de los proyectos y esperanzas que las mujeres habían incorporado a sus itinerarios, ya iniciados o de futuro, las nuevas generaciones tenían cerca referencias femeninas plurales, apreciadas, deseables. Quizás a esas alturas del siglo, incluso podía faltar memoria y conciencia del esfuerzo, impedimentos $\mathrm{y}$, en algunos casos, hasta menosprecio, sufridos -también satisfacciones y éxito- por unas jóvenes que se empeñaron en roturar sendas cuando la sociedad se sorprendía, y hasta sospechaba, de comportamientos tan fuera de la lógica al uso, excéntricos para la mentalidad que las rodeaba.

El objetivo de estas páginas es volver a la trayectoria académica y profesional de algunas de las pioneras a que me estoy refiriendo, que bien nacieron, estudiaron o trabajaron, en la provincia de Cádiz. Mujeres, unas con nombres conocidos, sobre las que ya se ha escrito, otras más desconocidas. Sabemos bien que contribuir a la memoria colectiva fortalece la identidad, alienta expectativas y forma parte del derecho a la verdad que la población femenina merece, pues está más acostumbrada a escuchar carencias sobre sí misma que reconocimientos y logros.

En la metodología utilizada para investigar esta temática, he partido del planteamiento de varias hipótesis que no son nuevas, ya me han servido de señuelo siempre que focalizo aspectos concretos de un tema general, el del acceso de mujeres a estudios superiores y al ejercicio de profesiones cualificadas. El punto de partida ha sido que las mujeres estuvieron activas en muchos espacios sociales públicos antes de que decidieran darles entrada quienes actuaban en nombre del patriarcado; que necesitaron mucha voluntad y resiliencia para conseguirlo; y que en Cádiz hubo mujeres, como en otras provincias, que estudiaron para aplicar después sus conocimientos en un trabajo remunerado. He revisado las publicaciones sobre esta temática que aparecen reflejadas en las citas introducidas a lo largo del texto. Como investigación documental, he consultado y localizado datos, sobre todo en fuentes primarias de archivo y en la prensa y revistas de la época. La cantidad de información encontrada sobre cada una de estas mujeres es desigual, pero creo que mostrar algunas pistas puede convertirse en un hilo que aprovechen otras investigaciones para llegar a otras fuentes y a nuevos resultados.

Seleccionar, organizar y contrastar el conjunto de informaciones, y utilizar el género como una categoría de análisis para situarlas en su contexto con perspectiva histórica, ha sido el 
camino metodológico que me ha conducido a la redacción de este trabajo; al acercamiento a hechos históricos que merecen mantenerse vivos en una historia de las mujeres a la que llevan tiempo contribuyendo tantas investigaciones con el objetivo de que sea mejor conocida y más transmitida. Difundirla es una responsabilidad que nos incumbe y un acto de justicia, tanto a ellas como a las generaciones jóvenes.

\section{Cultura y enseñanza en la provincia de Cádiz}

La relación de las mujeres con la cultura tiene en Cádiz una historia sobre la que no faltan estudios y publicaciones. Cultura entendida no sólo en su sentido más literal de conocimiento y de arte, sino también de manifestaciones que, en el contexto del siglo XIX, significaban reivindicación de una ciudadanía no adjudicada: implicación en causas políticas, asociacionismo, filantropía, etc. Algunas bien conocidas del Cádiz ilustrado, como María Ana de Arteaga (San Fernando, 1708-Cádiz, 1783), fundadora de escuelas de niñas en San Fernando y Cádiz. María del Rosario Cepeda (Cádiz, 1756-Madrid, 1816), niña premiada por sus conocimientos y activa joven ilustrada (Azcárate, 2000) o María Josefa de la Piedra (Cádiz, 1775-Sanlúcar de Barrameda, 1858), botanófila y colaboradora del Jardín Botánico de Madrid (Barras, 1945, pp. 38-39, Aedo, 2013). Muchas más en el siglo XIX, entre ellas Frasquita Larrea (Cádiz, 1775-Puerto de Santa María, 1838) escritora, con un salón de tertulia literaria y política en su casa (Fernández, 2003), Patrocinio de Biedma y La Moneda (Begíjar, Jaén, 1848-Cádiz, 1927) escritora, poetisa y periodista (Jiménez, 1984. Perea, 2004), pintoras (Triviño, 2011) como Victoria Martín Barhié (17941869), o fourieristas (Espigado, 2008; Pro, 2014).

Mujeres que actuaron sin esperar a verse incorporadas explícitamente en el ordenamiento jurídico de los derechos de ciudadanía, donde, por la mentalidad de los autores y el lenguaje utilizado, no se veían reflejadas. Pero el legado de una experiencia liberalconstitucionalista, las ideas de ida y vuelta que los puertos marítimos de la capital y de Algeciras favorecían, la población extranjera -familias afincadas y transeúntes-, vinculada fundamentalmente a la producción bodeguera y a la colonia inglesa de Gibraltar, las estancias más o menos estables de militares de diferentes lugares, y otras situaciones que podrían reseñarse, frenaban la intensidad de una estricta ortodoxia, del ethos cultural omnipresente, al menos en varias zonas de la provincia. En torno a la Revolución septembrina, de breve duración pero de potentes apremios de libertad, nuevas disposiciones legales despertaron expectativas de cambio, también en las mujeres; normativas, como dice la profesora Gloria Espigado que, orientadas al disfrute individual de los derechos ciudadanos, "propiciaron lecturas inesperadas, abiertas y comprensivas de los derechos femeninos que se debatían en algunos ámbitos, y que se tradujeron en actuaciones femeninas decididas a ocupar espacios inéditos y de enorme significación desde el punto de vista de la conquista de la ciudadanía" (Espigado, 2020, p.187).

Entre esos espacios inéditos ocupados, el de la enseñanza superior y universitaria, como alumnas primero, como profesionales después. No en vano, una de las prerrogativas propias de la ciudadanía era la instrucción pública ofrecida en un sistema escolar que comenzó a diseñarse en la Constitución de 1812, pasó por diversas concreciones en su desarrollo y fue aprobado en la Ley de Instrucción Pública de 1857. Su estructura estaba dividida en tres niveles, con voluntad de acoger a grupos de población estudiantil diferentes: la primera 
enseñanza, para alfabetizar a toda la población; la segunda enseñanza dirigida a las clases medias, impulsoras de progreso socioeconómico; y la universidad donde se formaba la clase dirigente, profesionales cualificados al servicio de las funciones asumidas por el Estado. Unas asignaciones que, como planteamiento ideológico y organizativo, limitaban la presencia femenina al nivel primario. Sin necesidad de prohibir, marcaban un terreno de juego ciudadano solo para hombres, no sospechando que pronto iba a ser frecuentada también por mujeres.

Recibir a las niñas en un aula escolar no fue fácil en el Cádiz del siglo XIX, lo mismo que en otras provincias españolas. El escaso cumplimiento municipal, en quién recayó el deber de crear y sostener escuelas y profesorado, mantuvo la escolarización en niveles bajos a pesar de las aportaciones de otros organismos como, por ejemplo, la Junta de Damas, la Casa de Misericordia, los colegios religiosos -privados y de carácter benéfico-, o las maestras particulares. Sin embargo, aun no disponiendo de una red escolar primaria suficiente para acoger a toda la infancia femenina, la proporción de mujeres alfabetizadas se mantuvo en Cádiz en mejores niveles que el de otras muchas provincias (Espigado, 1996, p.60).

La apertura de la Escuela Normal Superior de Maestras en 1857 significó disponer de un centro cuyo programa de estudios, elemental y superior -éste segundo suprimido de 1862 a 1881-, ampliaba el bagaje de conocimientos disponibles para las mujeres. Porque, más allá de formar a futuras maestras, acudían otras jóvenes con la única finalidad de completar la cultura recibida en la enseñanza primaria; por gusto de instruirse, saber, conocer. Jóvenes que demostraban con esta conducta interés por el estudio y unas expectativas de aprendizaje centradas más en adquirir conocimientos eruditos que en destrezas de utilidad doméstica o de lucimiento en sociedad. Pero que pudieron ser motivo de comentarios, debates a favor o en contra y, desde luego, referencia para otras, al verlas desplazarse camino de la Escuela Normal, organizar su rutina diaria reservando un espacio al estudio, mantener conversaciones en su entorno familiar o social cuya presencia introducía cuestiones menos habituales, las relacionadas con asignaturas, profesoras, motivos de la decisión tomada o porvenir al que aspiraban.

Y las profesoras normalistas estrenaban otra dedicación profesional, la de ser docentes de un centro de mayor nivel académico que la escuela de enseñanza primaria; realidad favorecida por la paulatina creación de Escuelas Normales en las distintas capitales de provincia durante la segunda mitad del siglo XIX. A la autoridad que se otorgaba a todas las maestras, unían ahora el hecho de ser formadoras de futuras maestras, docentes de alumnas que habían de recibir una formación más exigente, culturalmente más amplia y personalmente estimulante.

\section{Estudiantes y profesionales}

Centrándonos ahora en la enseñanza universitaria, comenzaré por las tres primeras que, con diferente finalidad, entran en la Facultad de Medicina de Cádiz. Antonia Monreal Andrés (Cádiz, 1872), inauguró con cierta temeridad el acudir en septiembre de 1889 a matricularse; solo un año antes se había reconocido a las mujeres, de manera explícita, el derecho a estudiar en la universidad, pero si deseaban asistir a las clases, tenían que contar 
con un permiso especial. Así lo tramita en una instancia de doce de septiembre dirigida al Decano de la Facultad en la que "interesándole continuar sus estudios en la carrera de Medicina y conviniendo asistir a las clases como alumna oficial para aprovechar la enseñanza completa de la misma [...]. Suplica a V.I. se digne admitir la matrícula oficial de esa Escuela, previo y conforme lo dispuesto, en que debe informar V.I. sobre las condiciones de la interesada. Es favor que no dudo alcanzar [...]" (AUCA, 1889). Quería aprender en las clases impartidas por los profesores, no prepararse las asignaturas fuera de las aulas, solo con libros. Desconocemos aún en qué condiciones de distancia de sus compañeros pudo asistir a las clases de las diferentes asignaturas, pero cumplió el requisito de ser autorizada para ello.

Con Antonia se matricula una compañera de bachillerato, mayor que ella, Ana Carvia Bernal (Cádiz, 1865-Valencia, 1941), que no llega a examinarse; la situación económica familiar, sus veinticuatro años de edad y los que exigía la carrera, más el compromiso adquirido en la actividad del movimiento librepensador, se interponen a su deseo. Aunque seguramente favoreció una mayor dedicación a la logia masónica gaditana de la que formaba parte y, en 1895, a la fundación, junto a su hermana Amalia, de la logia "Hijas de la Regeneración” (Almisas, 2020, pp. 4-6; López García, pp. 203-205).

Volviendo a Antonia Monreal, su expediente académico habla del excelente rendimiento obtenido: veintiún sobresalientes y cinco notables; también en el examen del Grado de Bachillerato la calificación había sido de sobresaliente. Durante los años de Facultad, la prensa local fue destacando esos éxitos en los exámenes, con los que "viene adornando su brillantísima hoja de estudios [...] y su reconocido talento y aplicación", animándola con un "tenga la seguridad de que con su constante aplicación hoy, y su honroso título mañana, compensará los sacrificios que su larga carrera representa" (S/A, 1893, p. 3). El examen de grado, final de carrera, lo realiza el 13 de febrero de 1896, una vez más con sobresaliente (AUCA, 1895). Aunque no sabemos si llegaron a coincidir en algún aula o pasillos de la Facultad, sí tuvo que saber que otras dos alumnas frecuentaron aquel espacio. En 18911892 Gertrudis Martínez Otero cursó el año preparatorio, y en 1894 una mujer nacida en Cuba, María Asunción Menéndez de Luarca (Guanabacoa, La Habana, 1858), había convalidado el título de medicina (Flecha, 1996).

Es probable que Antonia abriera consulta enseguida, aunque no he localizado publicidad de la misma hasta mayo de 1897 cuando, durante diez días no consecutivos, El Guadalete inserta el anuncio: “Antonia Monreal, Licenciada en Medicina y Cirugía. Especialista en partos y enfermedades de la mujer y niños, José del Toro, 23. Cádiz. Consultas diarias de 2 a 4. Gratis a los pobres Lunes, Miércoles y Viernes" (S/A, 1897). Este dedicar una parte de las consultas a personas pobres era una práctica, si no general, al menos frecuente entre la clase médica. Anteriores licenciadas en medicina lo hacían, y también muchas de las primeras décadas del siglo XX; eran años donde no existía un sistema de protección sanitaria generalizado.

Antonia se casa a finales de 1899 o principios de 1900 (S/A, 1899, p. 2) con el médico de Gibraltar Abelardo Triay Hernández -había estudiado la carrera en Cádiz entre 1871 y 1878-, cirujano en el Hospital de la Viruela, con consulta privada, y desde 1888 nombrado por el Gobierno para asistir a la clase de indigentes en la ciudad (Gibraltar, 1919, pp. 38- 
40). El mismo año 1900 solicita el alta como médica presentando los documentos de su licenciatura en Medicina y Cirugía. El Secretario Colonial consulta si, legalmente, había obstáculos para aceptar y el Fiscal General de Gibraltar responde que en la Ordenanza Médica local no figuraba nada sobre restringir la medicina a los hombres, y que la solicitud estaba debidamente avalada (More medical). Por lo tanto, parece que siguió ejerciendo la medicina, aunque no dispongamos ahora de más información.

\section{"Es muy largo de contar"}

Una estudiante de medicina que va a conseguir una especial relevancia en la ciudad es María del Mar Terrones Villanueva (Orce, Granada, 1892-Vigo, 1980). Pertenece a una familia dedicada a la enseñanza; maestro su padre, Manuel Terrones del Pino (Granada, 1860), maestras dos tías paternas, María Nieves y Concepción (Brácana, Granada), maestras sus hermanas Concepción (Baza, 1896), Margarita (Padul), María Consuelo (Baza, 1902) y María del Pilar; maestra iba a ser su hermana pequeña, Matilde, estudiante de la Academia Teresiana de Linares, pero fallecida en junio de 1915. También ella cursó la carrera de magisterio en la Escuela Normal Central de Maestras con un certificado de estudios de sobresalientes y premios; uno de ellos el de obtención gratuita del título de Maestra Elemental, "después de brillantes ejercicios" en las oposiciones "verificadas en la Escuela Normal Central de Maestras” en septiembre de 1907 (Gaceta, 1907, p. 645).

Todas las hermanas, en la convivencia con su padre, desarrollaron una vocación pedagógica y un hacer innovador que él reflejaba en todas las escuelas donde ejerció como maestro y en las que recibió felicitaciones y premios: Orce, Baza, Motril, Madrid, San Fernando y Cádiz. Que igualmente cultivaron durante un tiempo con la pedagogía povedana en su relación con las Academias Teresianas, y que María del Mar reflejaría en la actividad posterior.

Durante la estancia familiar en Madrid, María del Mar se matricula en el bachillerato del Instituto Cardenal Cisneros; lo realiza en dos cursos académicos, 1908-1909 y 1909-1910, obteniendo diecinueve sobresalientes -siete con premio-, dos notables y tres aprobados. Se le exime de las asignaturas de Religión y Gimnasia, cursadas en la Escuela Normal. En 1908 presenta un trabajo al Certamen Pedagógico organizado por los alumnos de la Escuela Normal Superior de Maestros de Alicante, y obtiene el "Premio de D. Aureliano Abenza, Da María del Mar Terrones y Villanueva, de Madrid” (S/A, 1908a, p. 1393). Aureliano Abenza era un profesor de aquella Escuela Normal que patrocinaba uno de los premios para el tema: "Papel que corresponde a Pestalozzi en la historia de la educación", y consistente en "Un objeto de lujo" (S/A, 1908b, p. 1)

El 28 de septiembre de 1910 hace los exámenes de Grado, de nuevo con sobresaliente y Premio extraordinario en la sección de Ciencias -la prensa recoge la noticia (S/A, 1910, p. 3)-, y el día 30 se matricula en el Curso preparatorio correspondiente a la Facultad de Ciencias Exactas, Químicas y Naturales de la Universidad de Sevilla -un primer año común para todas las carreras de Ciencias-, que se podía cursar en la Facultad de Medicina de Cádiz. Como había solicitado una plaza de maestra interina, en junio de 1911 es nombrada para una escuela de párvulos de Jerez de la Frontera (S/A, 1911a, p. 1026), cambiada unos días después a otra de Sanlúcar de Barrameda, pero cesa en el cargo en el mes de julio 
(S/A, 1911b, p. 2). Además, en el mes de septiembre supera con sobresaliente en la Escuela de Comercio el preceptivo examen de lengua alemana en la carrera de Medicina. Con todo este llamativo bagaje de estudiante excelente ha llegado a vivir a San Fernando, Cádiz, donde se había trasladado su padre y toda la familia.

En la Facultad de Medicina gestiona que los sobresalientes y premio extraordinario en el grado de bachillero, la eximan del pago de derechos de las cuatro asignaturas del curso preparatorio, como harán curso tras curso ante los mismos resultados en casi todas las asignaturas. En 1911, matrícula oficial de tres asignaturas, el curso siguiente matrícula no oficial de otras tres. En 1913-1914 vuelve a matrícula oficial y en el mes de febrero, el día 10, firma haber recibido el "Título de Alumno interno numerario (sic) con destino a las Clínicas generales de esta Facultad". Había obtenido el n. ${ }^{\circ} 1$ en las oposiciones para cubrir esa plaza (S/A, 1914a, p. 1) donde comenzó su práctica en la actividad médica, solo interrumpida durante ocho días de noviembre,

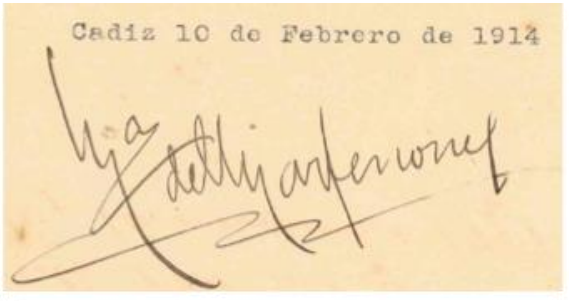

Firma del Recibí el Título de Alumno Interno por encontrarse "algo delicada de salud, lo que la imposibilita asistir al hospital clínico a cumplir los deberes de su cargo”. (AUCA, 1910)

En el mes de abril de 1915 renuncia a la matrícula oficial de seis asignaturas de "clase ordinaria", no "de honor", para cambiarla a "no oficial" y así le es concedido previo acuerdo de los catedráticos correspondientes. Nueva matrícula no oficial el 31 de agosto, ya "de honor" en las cuatro asignaturas, y cuatro sobresalientes en el examen del 30 de septiembre. Este curso de 1914-1915 se le acumulan las responsabilidades que, seguramente, justifican tal cambio. En septiembre de 1914 se había estrenado como directora de la Academia Teresiana de Cádiz y en diciembre como profesora de Educación Física de la Escuela Normal (AGA, 1914).

Una entrevista en la que participa cuando cursaba en Madrid las asignaturas del doctorado y asistía al Hospital del Niño Jesús, nos permite saber las motivaciones y el ambiente que vivió en la Facultad de Medicina de Cádiz. Sobre la razón de estudiar medicina dijo: "Arranca de mi niñez. Cuando yo tenía cuatro o cinco años, al jugar con mis compañeras de la misma edad, yo era siempre el médico que las asistía". La figura del médico de alguno de los pueblos en los que ejercía su padre debió despertarle el deseo de ese trabajo; lo que parece reflejarse en otra de las respuestas: "Me vi en la necesidad de ser un poco muchacho y hacer una hombrada". El periodista se interesa por sus años de estudio en Cádiz, por cómo los vivió en la Facultad, y ella, quizás no queriendo entrar en el tema respondió: "Es muy largo de contar". Pero ante la insistencia del entrevistador hace una reflexión de carácter general: "Tiene usted que tener en cuenta el ambiente creado a las mujeres españolas, el miedo propio a que las fuerzas no basten; la lucha que significa no solo el estudio, sino la situación entre los compañeros". Lo que se esperaba de las mujeres y la idea de incapacidad que se les transmitía, las hacía dudar de la conveniencia de sus decisiones y de su capacidad para el estudio.

Compartir con los compañeros aulas y pasillos, pensados entonces como espacios masculinos, entorpecía las dinámicas de relación, si bien afirmaba no haber tenido 
dificultad, aludía a "lo escabroso de la carrera, en qué, por los especiales conocimientos que hay que adquirir, una mujer necesita un gran tacto y un gran respeto". Quiso distender un poco el clima comentando: "Los hombres son ustedes tan expresivos, y ¡en Andalucía!... Las mujeres andaluzas, que no vemos mal un piropo, a veces, en mi caso, preferiríamos el silencio, que se acuerda mejor con nuestra situación anómala”. Aflora de nuevo aquí el sentimiento de estar ocupando un lugar indebidamente y la conciencia de ser protagonistas de una situación irregular y extraña por no ser hombres. A la vez, con la incómoda actitud que las estudiantes se veían obligadas a mantener: "Hay que aislarse, hay que imponerse un régimen de silencio, y agradecer la cortesía que se nos hace". Pero su experiencia con los compañeros había sido positiva pues "me concedieron en todo momento el respeto que les pedí, y en el que supe mantenerme siempre". El periodista desea más e insiste, "Pero el respeto sin cordialidad..." Y María del Mar reitera: "Ya le hablé de aislamiento. En España, aún, la mujer no puede ser camarada de su compañero. Se mantiene una como en visita. Una visita de 6 años, un poco larga y un poco enojosa también. La conversación, así, se sitúa siempre sobre el estudio y cuándo se acaba el tema se acaba la conversación" (Aguilar, 1917, p. 2). Unas declaraciones que nos permiten conocer de primera mano cuál era la atmósfera externa, y la interna personal, en la que discurrió la experiencia universitaria de aquellas jóvenes.

Pero ni estas condiciones ni la dedicación y rendimiento académico que obtenía, fueron óbice para su participación en distintas colaboraciones y compromisos. En diciembre de 1911 es admitida como socia de mérito en la Sociedad de la Prensa de Cádiz, donde la habían precedido Patrocinio de Biedma y Emma Calderón, ya "que a juicio de la Asociación lo merecían por los servicios prestados a las misma" (Salaverry, 2003, p. 62; Ruiz, 2011, p. 248); se publica una foto que ocupaba toda la portada de la revista Diana con un pie, junto a su nombre: "Maestra de primera enseñanza; Alumna oficial de la Facultad de Medicina de Cádiz, y Socio (sic) de Honor del Centro Escolar Gaditano" (S/A, 1911c). El Centro Escolar Gaditano creado ese mismo año, era una asociación que buscaba la fraternidad entre los estudiantes; tenía como finalidad social "estrechar los lazos de unión de amistad y compañerismo entre los estudiantes de los diversos centros de enseñanza de esta capital" (Rodríguez, 2016, p. 72). La foto procedía de la Revista Escolar, una publicación creada en mayo de 1905 y dirigida por el maestro Ildefonso Yáñez "para defensa de la clase a que su título se refiere" (S/A, 1905, p. 2).

Y en el Diario de Cádiz publica un artículo uniéndose al movimiento estudiantil universitario en contra de la versión difundida por la escritora Rosario de Acuña sobre un suceso protagonizado por estudiantes madrileños. Se hizo eco de este artículo, y del escrito por Emma Calderón sobre el mismo tema, no solo la prensa local, también la madrileña. En el periódico La Época podemos leer "que entusiasmaron a los estudiantes" (S/A, 1911d, p. 2).

Aún no había cumplido veinte años, su estancia en la provincia era aún reciente y, sin embargo, sus cualidades la habían convertido enseguida en una persona conocida y valorada. Y mientras estudia la carrera, su actividad, que la prensa local sigue y publica, continúa siendo intensa. En marzo de 1912 ocupó un lugar en el estrado de la sesión de clausura de las Conferencias Escolares (S/A, 1912a, p. 1); en abril asistió a un acto de recibimiento académico en la Real Academia Hispano-Americana de Cádiz, junto a 
Patrocinio Biedma, Emma Calderón, la directora y la profesora de Ciencias de la Escuela Normal y otras. (S/A, 1912b, p. 1); en mayo la Revista Diana publica un texto dedicado a María de Mar en el que es comparada con las sirenas: "Una émula de las sirenas por sus bellezas y encantos y por su ingenio con la pluma, la joven y hábil escritora a quien dedico esos torpes renglones y los que creo aceptará gustosa" (Honahilag, 1912, p. 4); en junio se suma a las adhesiones que recibe el Congreso de Periodistas Español organizada por la Asociación Gaditana de la Prensa (S/A, 1912c, p. 3), junto a Emilia Pardo Bazán, Patrocinio de Biedma y Emma Calderón "todas ventajosamente conocidas en el mundo intelectual" (S/A, 1912d, p. 3).

El 10 de noviembre pronuncia el discurso inaugural de la entrega de los premios del Círculo de Artes y Oficios de San Fernando, elogiando la labor que desempeñaba en bien de la educación y lo que significaba para el porvenir de la sociedad: "Esta es una gran fiesta de amor. Fiesta de fe; sí, de fe en el porvenir, en el ideal de sus organizadores. [...] Con la bandera de la cultura y el escudo de la enseñanza. [...] La cultura popular moderna no es la escuela antigua [...] es eminentemente educativa, llena de amplios ideales y cuajada de nobles pensamientos". (El corresponsal, 1912, pp. 1-2). En este discurso, donde no faltan las frases enfáticas y las alabanzas encendidas, manifiesta su confianza en la educación, en la defensa que garantiza frente a la vida -es bandera y escudo- proponiendo un concepto de cultura popular más educativa -de ideales y valores-, que instructiva.

Al día siguiente, el 11 de noviembre, asiste a una velada académica en la Real Academia Hispano-Americana de Cádiz donde es citada por el conferenciante por sus "brillantes producciones" (S/A, 1912e, p. 3). Y para cerrar el año, en el mes de diciembre, se organiza un banquete en honor, "de la bella e ilustrada... con motivo del gran éxito que tuvo su último discurso, pronunciado en el reparto de premios del Centro Obrero". Acto en el que María de Mar "contestó con un discurso brillantísimo, siendo ovacionada". (S/A, 1912f, p. $3)$.

Actividades que se van sucediendo y que pasan a ser más académicas a medida que transcurre 1913 y, sobre todo 1914. Implicada también en la vida de la Facultad, asiste al banquete que los alumnos de Medicina ofrecen a uno de sus profesores con motivo de su elección como concejal (S/A, 1913, p. 2). Y el conocimiento médico que fue adquiriendo le hace tomar conciencia de la importancia de enfermeras bien formadas, objetivo con el que organiza en el Centro Protector de Obreras unas clases preparatorias para los exámenes de aspirantes a enfermeras que comenzarían en el mes de abril. (S/A, 1914b, p. 1). Así contribuía, igualmente, a que más mujeres jóvenes decidieran entrar en este campo.

En agosto de 1914 comienza a aparecer en la prensa gaditana la información sobre la apertura en la ciudad de la Academia Teresiana, figurando como "Directora Técnica la Srta. María del Mar Terrones. (S/A, 1914c, p. 3). A través del profesor de Religión de la Escuela Normal, María del Mar ha tenido noticia de las iniciativas que Pedro Poveda, un sacerdote ocupado en diferentes proyectos educativos, que desarrollaba en varias ciudades españolas, La propuesta concreta es abrir una Academia-Internado que apoyara la preparación recibida por las Maestras en la Escuela Normal y facilitara un alojamiento de estilo familiar y culturalmente alentador a las que se desplazaran desde otras localidades de la provincia. Con clase de párvulos, de primera enseñanza y de segunda enseñanza para las niñas que 
estudiaran el bachillerato. En el intercambio de comunicación mantenido en este tiempo, María del Mar se ilusiona con una obra centrada en la promoción académica y profesional de las mujeres, pedagógicamente innovadora y orientada por los valores del evangelio (Ruiz Guerrero, 2011, pp. 251-256). La experiencia fue corta, de un curso académico pues, aunque cuenta con el apoyo de sus hermanas maestras y de su madre, María Mar pasará muchas temporadas en Madrid cursando las asignaturas del doctorado durante tres cursos académicos. Una de las hermanas Margarita se incorporó como profesora a la Academia Teresiana de Linares en diciembre de 1914 y la más pequeña, Matilde, fue allí a estudiar magisterio. En este viaje fueron acompañadas por María del Mar y la prensa de Linares lo recogió en sus páginas: "La notable institución de enseñanza [...] que debemos a la intuición pedagógica del Padre Poveda [...] ha tenido la fortuna de aumentar su profesorado con una joven cultísima, de noble abolengo intelectual: la señorita Margarita Terrones y Villanueva, [...]. La acompaña su



Margarita Terrones. (Institución Teresiana, Madrid. A.H.I.T. II/a.g.5/2.4) hermana, María del Mar, directora de la Academia Teresiana de Cádiz”. (cita en Velázquez, 1996, p. 180, tomada del periódico linarense La Unión, 18 diciembre 1914).

En la Residencia Teresiana de Madrid, inaugurada en marzo de 1914 María del Mar va a convivir con las primeras estudiantes que se alojan en ella, entonces alumnas de la Escuela Superior del Magisterio y de otros centros de Enseñanza Superior. Durante el primer curso de funcionamiento el grupo llegó a ser de quince alumnas, la directora Mariana Ruiz Vallecillo y su madre que llevaba la administración: María del Carmen del Amo Ramos, de Córdoba; Isabel del Castillo Arista, Concepción Montoro Cerezo y Manuela Ortega Povedano, de Linares; Herminia Castrillón Subirats, de Santiago; Carmen Cuesta del Muro, de Palencia; Manuela García Luquero y Magdalena Martín-Ayuso, de Oviedo; Victoria Kent Siano y Victoria Montiel Vargas, de Málaga; Mariana y Elvira Ruiz Vallecillo y su madre, Ana Vallecillo, de Ronda; María del Mar Terrones Villanueva, de Cádiz; Cándida Valenzuela Pastor, de Jaén; Matilde Gómez Rodríguez, de Soria; y Francisca López Gutiérrez, de León. En la foto siguiente podemos ver a la mayoría de aquel grupo de 19141915.

Durante varios años estudia algunas de las asignaturas se matricula en asignaturas del periodo del doctorado, pero de la consulta de los archivos que corresponden a este trámite se deduce que no llegaría a leer la Tesis. Todavía el siete de enero de 1920 vuelve a hacer matrícula de dos asignaturas en enseñanza no oficial (AUCM, 1915), tres meses antes de su boda, el siete de abril, con un hombre de alto nivel económico, Teodoro Vega Díaz, propietario, consejero del Banco Hispano Americano, exdiputado provincial de Asturias y con negocios en España y Filipinas. Un matrimonio que la lleva a cambiar la dedicación de su tiempo, pero no su inquietud educativa y de actuaciones públicas de las mujeres. Se une al Lyceum Club Femenino, creado en España en 1926 tomando como modelo el londinense, en cuyo listado de socias aparece como "Vega, María del Mar Terrones de (Rosales, 58)" (Aguilera, 2011, p. 88); asociación de mujeres que exigía ciertos requisitos de cultura y de protagonismo social para incorporarse a él, los cuales cumplía ella con creces. Además, 
estaba presidido por María de Maeztu, a la que conocía del breve tiempo que pasó en Cádiz como profesora de la Escuela Normal. Un espacio que ofrecía a mujeres de un estatus sociocultural y económico alto, poder ocupar una parte de su tiempo en actividades culturales, intercambio de ideas e inquietudes, compartir con las que estaban ejerciendo una profesión.

María del Mar pensó mucho en cómo debía ser la educación de sus dos hijas y dos hijos, y no encontrando en Madrid un centro que respondiera a sus expectativas, decidió crearlo en 1928 con las condiciones que buscaba. Esfuerzo económico que podía permitirse, invertir en la que llamó Escuela Internacional Española, donde el aprendizaje de idiomas era el eje de toda la enseñanza. Eligió para dirigirla al institucionista José Castillejo y con el respaldo jurídico de la Sociedad de Educación Internacional presidida por el poeta Pedro Salinas y ella como vicepresidenta (Pozo, 2013, pp. 225-226). Después de la guerra pasó a ser la presidenta y, en diciembre de 1953, disolvió esta Sociedad (R. 1954, p. 53).

Otra prueba de su interés por la educación la vemos en su cercanía al Colegio Nacional de Ciegos, de cuyo Comité de Tutela Social terminó siendo Vocal, "teniendo en cuenta las elevadas condiciones que concurren” en las personas nombradas (GM, 1935, p. 1319).

\section{Médicas, farmacéuticas, maestras}

Médica también, Adelaida Navarro Patiño (Orgaz, Toledo, 1907-Madrid, 1977), realiza el bachillerato en el Instituto de Segunda Enseñanza de Badajoz -figura con el nombre de Adela- y la carrera de Medicina en la Facultad de Cádiz. Se matricula desde el curso 19251926 al de 1930-1931, y en su certificado de estudios destaca una mayoría de sobresalientes. En la orla de su promoción es la única licenciada que aparece, junto a cincuenta y dos compañeros y catorce profesores. Cuando cursaba las últimas asignaturas de la carrera se presentó a "los ejercicios correspondientes" para un puesto en la Inspección Municipal de Sanidad obteniendo una de las veinticinco plazas que daban derecho al "Título de Inspectora Municipal de Sanidad". El resto de sus compañeros en este grupo fueron igualmente todos hombres. (S/A, 1930, p. 2).

Adelaida vive en Cádiz con una hermana, Mari Cruz, que trabaja en una plaza de Auxiliar de la Delegación de Hacienda; había estudiado el bachillerato en Badajoz y ganado las oposiciones al Cuerpo general de la administración de Hacienda; un hermano, Ángel, estudia en la Facultad de Medicina y aficionado a la poesía, la cultivaba en la peña literaria que se reunía en el Hotel Atlántico. El padre, Ángel Navarro Sánchez (Guadalajara, 1860), era maestro en propiedad desde 1887; nombrado en 1905 para la escuela de niños de Orgaz, localidad en la que nace Adelaida, y en 1916 para una escuela de Badajoz, en cuyo Instituto estudia el bachillerato. También la madre, Adela Patino Sánchez (Toledo, 1875), ejercía como maestra en propiedad desde 1895. Diez años después su destino es el pueblo toledano de Guadamur y en 1907 la escuela de niñas de Orgaz, "en virtud del derecho preferente establecido para los maestros consortes" (GM, 1907, p. 279); más tarde es maestra en Badajoz y desde 1933 en la clase de párvulos de la escuela "Joaquín Costa" de Cádiz (GM, 1933, 871).

En abril de 1932 traslada el expediente a la Universidad de Madrid con la intención de 
cursar el doctorado, aunque no parece que lo hiciera. En cambio, en 1933 se presenta a un concurso de la Escuela Nacional de Puericultura para una plaza de alumna como médica puericultora, y ejercerá dentro del cuerpo Médico de Asistencia domiciliaria. En 1934 abona la mitad del coste del título viviendo en Riotinto, Huelva, y la otra mitad en marzo de 1940 (AUCA, 1924). Con el título ya emitido, en 1941 entra a formar parte del Colegio Oficial de Médicos de Cádiz. Sobre el proceso de depuración de Adelaida, por el que fue obligatorio pasar como consecuencia de la guerra civil, el dato conocido es incompleto. Únicamente la reseña que figura en las Actas del Colegio Oficial de Médicos de Cádiz diciendo: "Navarro Patiño, Adelaida (Cádiz). Pendiente de depuración "por no haberse recibido todavía su certificado de depuración de Huelva" (1941)" (Cabrera, 2004, p. 412).

En 1942 se presenta al concurso de antigüedad y es nombrada para la plaza de Casasbuenas, Toledo (BOE, 1942, p. 5028), opositando dos años después para el acceso a la plaza en propiedad (BOE, 1944, p. 7325). Interrumpe este proceso porque en 1951 entra en la Compañía de las Hijas de la Caridad de San Vicente de Paúl, donde sería directora, primero de la Escuela de Enfermeras que tenían en el barrio de Carabanchel, y después en la del Hospital Clínico de Madrid. En 1958 participó en el Congreso de la Federación Católica de Instituciones Hospitalarias celebrado en Bruselas, que tuvo lugar en Bruselas (AHCM). En 1952 formó parte de la Comisión Central del Ministerio para Estudios de Enfermería, cuando se planteó unificar las carreras de Enfermera, Practicante y Matrona en la de Ayudante Técnico Sanitario (Hernández, 2006, p. 45).

Razón por la que en 1959 figuró en el listado de facultativos eliminados del Cuerpo de Médicos titulares al "haber dejado transcurrir más de diez años en situación de excedencia voluntaria sin reingresar al servicio activo" (BOE, 1959, p. 15161).

Considerando a Ceuta, como en aquel tiempo, estrechamente vinculada a la provincia de Ceuta, entre las médicas añadimos a Herminia Adrados Vicente (Ceuta-Madrid, 1969), hija de militar, que ejerció en Madrid la plaza de médica puericultora y pediatra del Estado, ganada por oposición en 1943. Había estudiado la carrera en Madrid, alojada en la Residencia de Señoritas durante los cursos de 1929 a 1933 (Vázquez, 2012, p. 3749) y finaliza la licenciatura en 1935. Publicó numerosos trabajos y tuvo intervenciones en Congresos, donde sus aportaciones pusieron de manifiesto una forma de crear ciencia y de difundirla a partir de los conocimientos que le estaba proporcionando su experiencia en la práctica del cuidado de la salud y del tratamiento de la enfermedad.

Una gaditana con carrera y ejercicio de la profesión en Cádiz fue María de los Dolores de Sarriá Guerrero (San Fernando, 1913). Con 26 años, título de bachiller y de enfermera, se matricula en la Facultad de Medicina el curso 1938-1939 en enseñanza no oficial; el 31 de agosto de 1939, para examinarse en la convocatoria extraordinaria de septiembre. En instancia al Rector de la Universidad de Sevilla solicita quedar exenta del examen de ingreso, amparándose en una Orden -ella dice Decreto- de 20 de agosto "concediendo exención de examen de ingreso en Universidad [...] a escolares militares o perseguidos durante la dominación marxista" (BOE, 1939, p. 4682). Aunque el lenguaje es masculino ella no duda en sentirse incluida en esas condiciones. ¿Qué motivos tiene para ello? Los datos que expone nos permiten conocer que esta enfermera había pertenecido "al Servicio de Guerra de Hospitales como Enfermera Diplomada y prestado sus servicios durante la 
Guerra durante dos años en los Hospitales de Sangre Musulmanes, en los de 1937 y 1938 , como Enfermera de las Salas de Operaciones del Hospital Militar de Ronda".

Había sido una de aquellas mujeres, muchas que, de acuerdo con suposición ideológica unas veces, y otras solo por la coincidencia geográfica donde vivieron el conflicto bélico provocado por el golpe de Estado, aportaron su trabajo y su conocimiento para mitigar el incomprensible sufrimiento generado; la mayoría no con las armas, sino curando, acompañando, dando esperanza en medio del horror exasperante. María Dolores, además de contar con los comprobantes que lo avalaban, exponía lo siguiente: "Que en vista de estos servicios militares a las fuerzas indígenas S.A.I. el Jalifa le ha concedido "la Medalla de la Medahuia". Aceptada su solicitud, se matricula de cinco asignaturas y se examina de tres en las que obtiene aprobado. Los cursos siguientes, hasta 1942-1943, estudia con matrícula oficial y las calificaciones van mejorando, con varios sobresalientes y premios. (AUCA, 1939). El Título, le fue expedido con fecha de 15 de noviembre de 1944.

Dos décadas antes, la hermana mayor, Josefa, finalizado el bachillerato con dieciséis años en el Instituto de Cádiz, se había matriculado de una asignatura del curso preparatorio de la Facultad de Ciencias (Cádiz), en agosto de 1917, en la enseñanza no oficial del curso 19161917; la supera con un aprobado. Tres asignaturas más en el curso siguiente, con nota de sobresaliente en junio; y de la última, que completaba el preparatorio, se examina en la Universidad de Granada en la convocatoria de septiembre. En esa Universidad estudia la carrera de Farmacia de 1918 a 1921 (AUG, 1917) obteniendo el Título el 13 de marzo de 1922. Dirigió una Farmacia en su ciudad de origen, San Fernando. Otras dos hermanas, Carmen (San Fernando, 1902) y Teresa, estudiaron en la Escuela Normal; Carmen aprobó en 1933 los Cursillos de Selección para el magisterio nacional. El padre era médico, teniente coronel Jefe de Sanidad de la Armada.

Con estos precedentes, a María Dolores no le resultó difícil superar el proceso de depuración tras la guerra civil; el libro de Actas del Colegio Oficial de Médicos de Cádiz recoge: "Depuración favorable como persona adicta al Glorioso Movimiento Nacional" (Cabrera, 2004, p. 416). Ejerció como pediatra y puericultora, atendiendo la consulta privada en que había iniciado su itinerario profesional $\mathrm{y}$, más tarde, como médica del seguro obligatorio de enfermedad hasta su jubilación. De esta manera pudo ser una referencia precursora de otras muchas en una actividad de saberes cultivados siempre por mujeres, con ese tipo de conocimiento, he dicho antes, proporcionado por la experiencia y transmitido de mujer a mujer a lo largo de los siglos, pero desvalorizado por la ciencia médica, la que estas licenciadas tuvieron que adquirir en las aulas universitarias.

Estudiantes en la Facultad de Medicina de Cádiz, fueron Francisca Medina Verdeja (Málaga, 1891-Madrid, 1977), sobre la que contamos con el completísimo artículo del profesor de la Universidad de Málaga Víctor M. Heredia Flores (2021, pp. 14-31); la maestra catalana Magdalena Cirera y Paula que, dentro de su complicado itinerario en el ejercicio del magisterio, se matricula varios años en la Facultad, si bien su dedicación será de maestra en Málaga y Barcelona. o Lorenza Baena.

En la Universidad de Granada terminaron Farmacia, la que fue primera alumna de la Facultad Gertrudis Martínez (Sanlúcar de Barrameda, 1868-1965) en 1896, Manuela Alba

$\mathrm{N}^{\circ}$ 9, 2021. Página| 30 
Gómez (Tarifa, Cádiz) en 1921, Carmen y María Pilar Aranda Rubio (Ceuta, Cádiz) en 1925, Ma de los Milagros Tardío Viejo (Rota, Cádiz) en 1927. Y en la Universidad Central, Rosa Herrera Montenegro (Jerez de la Frontera, Cádiz, 1891-1977), con doble licenciatura pues se examina también de la rama de Ciencias Naturales; los planes de estudio de ambas compartían un grupo de asignaturas comunes. El bachillerato lo había realizado en el Instituto Cardenal Cisneros de 1904-1905 a 1914-1915, y la universidad de 1915 a 1919 (AHN, 1904). Cuando estaba finalizando la carrera formó parte del primer grupo universitario que se incorpora al Instituto-Escuela en la figura de Aspirantes al Magisterio Secundario, al crearse este centro en 1918. Es admitida en la sección de Matemáticas dirigida por el catedrático de la Universidad Central Julio Rey Pastor (Palacios, 1988, p. 108); cambia después a la sección de Ciencias Naturales. De 1923 a 1925 pasa a encargarse de la dirección de prácticas del Laboratorio de Química de la Residencia de Señoritas en el que había colaborado como auxiliar de su directora, la profesora norteamericana Mary Louise Foster. Fue becada por la JAE para estancias en Londres, París y Ginebra para investigar la enseñanza de la química (Magallón, 1998, pp. 324-325). En 1933 aprueba los Cursillos de Selección del profesorado de Segunda Enseñanza y ejerce unos años la docencia de Historia Natural y de Agricultura en los Institutos de Alcázar de San Juan, de Lérida y de Figueras, consecutivamente (Flecha, 2010, pp. 663). Abre también una Farmacia en la calle Francisco Silvela de Madrid, la cual permaneció cerrada durante los años de la guerra civil y hasta 1945, periodo de tiempo que Rosa Herrera se ausenta de Madrid. No deja de ejercer como profesora en Institutos de la zona del gobierno de la República, en Lérida y en Figueras (GR, 1938), para, finalmente, exiliarse en Francia. Circunstancias que llevaron al gobierno de Franco a incoar contra ella "expediente de responsabilidades políticas" (BOE, 1942). Pero, "a partir de 1945, consta en el Archivo del Colegio [Oficial de Farmacéuticos] su continuidad al frente de la citada oficina de farmacia” (García y Renáu, 2020, p. 47)

En Cádiz nacieron las hermanas Aguayo Balseiro, María de los Reyes (Cádiz, 1908) y María del Carmen (Cádiz, 1913-Madrid, 1999) donde su padre fue unos años profesor del Instituto de Segunda Enseñanza; trasladado a Madrid, estudian el bachillerato en el Instituto de San Isidro y la carrera de Filosofía y Letras en la Universidad Central. Carmen terminó la carrera en 1933 participando ese verano en el Crucero Universitario por el Mediterráneo (Poveda, 2014, p. 94) a bordo del Ciudad de Cádiz; cuarenta y cinco días visitando yacimientos arqueológicos de Túnez, Malta, Egipto, Palestina y Tierra Santa, Beirut, Damasco, Creta, Rodas, Grecia, Italia, y otros. Y en 1934 se estrena como profesora Ayudante de Instituto, ejerciendo en diferentes centros hasta su jubilación (Poveda, 2014, p. 432).

Maestras como Ángeles Gatica Rumazo, nacida en San Fernando en 1863 que, después de estudiar en la Escuela Normal de Cádiz y obtener una plaza en el magisterio nacional, estudia el bachillerato en los Institutos de Cádiz y de Sevilla, y solicita traslado a Madrid en cuya Universidad cursa la licenciatura en Filosofía y Letras y las asignaturas del doctorado. En varias ocasiones firma oposiciones para profesoras de Escuela Normal, pero no llega a obtener plaza. Como Carmen Abela Espinosa de los Monteros (Chiclana de la Frontera, Cádiz, 1875-Guadalajara, 1962) interesada y estudiosa de la infancia con limitaciones personales para el estudio y para la vida, a la que entonces se nombraba con el término de anormal; se centró especialmente en la atención a los sordomudos. Para ampliar su 
formación en este campo solicitó y recibió en 1920 una pensión de la JAE que le permitió visitar el Instituto Nacional de Sordomudos de París; allí, junto a Mercedes Rodrigo pudieron observar un centro de formación de profesorado con esa especialidad. Formación que en España se realizaba mediante un curso muy breve, llamado "Métodos y procedimientos para la enseñanza de los sordomudos", en el Colegio Nacional de Sordomudos, al no existir ninguna institución dedicada a ello (Herráiz, 1995, p. 153). En una obra de Teresa Marín leemos que ya había solicitado en años anteriores esta pensión, aportando como aval muchos trabajos y experiencias en este campo; cursos, investigaciones, trabajo directo, por ejemplo, en el Sanatorio Marítimo Nacional de Pedrosa, Santander. El tema de la solicitud fue "La situación de la pedagogía de anormales en los países europeos", concretando en los de Francia, Suiza y Bélgica. Una pensión concedida en 1920 y prorrogada para 1921. (Marín, 1991, pp. 17-20).

También merecen considerarse las trayectorias de las profesoras de Escuela Normal Encarnación de la Rigada, Carmen Oña Espert (Sanlúcar de Barrameda, 1864), licenciada en Filosofía y Letras, María Pilar Velasco Aranaz (Algeciras, 1883), y de las que ejercieron en la Normal de Cádiz; o de la licenciada en Filosofía y Letras y bibliotecaria Ana María Liaño Pacheco (Cádiz, 1906-Sevilla, 1963).

Pero me detengo en una Inspectora, Teresa Izquierdo Izcue (Castellón, 1886-Cádiz, 1955) que llega a Cádiz, a una ciudad donde va a discurrir toda su vida profesional, como Inspectora de Primera Enseñanza. Su nombramiento es del día 10 de julio de 1920 (GM, 1920, p. 270), y el 27 de ese mes toma posesión. La prensa, al dar cuenta del acto, comenta que "por lo que hemos podido apreciar en su carácter bondadoso, su interés por la enseñanza y su amor a la escuela es seguro que muy pronto tendrá la estima de sus subordinados con lo cual saldrá bien parada la enseñanza de las niñas y la educación de ésta, pues de esperar es que los consejos que de ella dimanen, serán de una orientación en todos los órdenes" (S/A, 1920, p. 3). Es la primera mujer que ejerce en la provincia gaditana una labor educativa contando con la buena formación recibida en la Escuela de Estudios Superiores del Magisterio y con la experiencia de haber sido previamente maestra. Pertenecía a la promoción de 1915-1918, Sección de Labores, en la que elaboró la Memoria fin de carrera sobre "Ligero apunte para el estudio de los trajes regionales de la provincia de Toledo, principalmente del tipo llamado de Lagarterana" bajo la dirección del catedrático Luis de Hoyos; un trabajo que este profesor presentó en un congreso internacional (Ortiz, 1987, p. 112) y reseñó en una de sus publicaciones (Hoyos, 1922, p. 96). Era el primer estudio que se realizaba sobre ese tema y fue muy valorado por las numerosas aportaciones que hacía. Cuando en junio de 1936 se constituye el Patronato y Comité del Museo del Pueblo Español, creado en julio de 1934 (GM, 1934, pp. 995-997), Teresa Izquierdo es nombrada patrona y correspondiente regional de este Museo en Cádiz (RE, 1935).

Su actividad en la Inspección refleja muy bien el interés por mejorar la enseñanza y poner a disposición de las niñas y niños recursos que mejoraran la labor educativa en las aulas. Enseguida organiza, con sus dos compañeros Inspectores, Cursillos de perfeccionamiento para el magisterio (S/A, 1921, p. 3; 1928, p. 3). En la Exposición de Labores de la Mujer promovida por la Escuela Normal de Maestras, "es una fervorosa propagandista de este proyecto que, sin olvidar su misión inspectora, labora con el mayor entusiasmo por atraer 
expositores, en un anhelo generoso digno, del más alto triunfo" (S/A, 1925a). Organiza Colonias Escolares (S/A 1925b). Abre Cantinas en varias escuelas (S/A, 1925c, pp. 1-2). Forma parte de la Comisión para celebrar en Cádiz el Centenario del pedagogo Pestalozzi (S/A, 1927, p. 1).

Cuando en los primeros meses de la guerra civil, las autoridades de la zona en que Cádiz estaba situada deciden el comienzo del curso escolar al menos en las escuelas primarias, se constituye la Gestora Provincial de Primera Enseñanza en la que figura Teresa Izquierdo, por elección del Gobernador Militar (Pettenghi, 2005, p. 28). Una de las órdenes que hubo de transmitir en febrero de 1937, es que la supresión de los carnavales suponía también la de la vacación acostumbrada: "Inspección provincial de primera enseñanza de Cádiz. Vista la orden dada por el excelentísimo señor gobernador de esta provincia, respecto a la supresión en el presente año de las fiestas de carnaval. La inspección de primera enseñanza hace saber a los señores maestros nacionales que dicha fiesta figura en el almanaque escolar, la vacación se considera suprimida. Cádiz, 6 de febrero de 1937. Teresa izquierdo" (S/A, 1937, p. 4).

En el proceso de depuración sufrido durante la guerra civil Teresa fue designada para formar parte de la Comisión depuradora de Cádiz. A este respecto, el profesor Pettenghi comenta de ella que trabajaba "en la Sección Administrativa de Primera Enseñanza, que entonces tenía su sede en la Aduana. Si bien era de ideología muy conservadora, no puede decirse que fuera afín a Falange" (Pettenghi, 2005, p. 56). Y en febrero de 1937, una Orden firmada por el Director General de Primera Enseñanza del gobierno de la República, acordó "la separación definitiva de sus cargos, con pérdida de todos los derechos que pudieran derivarse de su situación escalafonal", junto a otras diecisiete Inspectoras y cuarenta Inspectores (GR, 1937, pp. 625-626). Pero, como señala el Inspector de Educación de Andalucía Manuel Santander, después de 1939 las orientaciones educativas fueron muy diferentes a las anteriores, y así había que transmitírselas a las maestras. En este caso, reproduce un párrafo del Libro de Visitas de la Graduada n. 6 de Cádiz escrito por esta Inspectora: "En las visitas realizadas a la Graduada n. ${ }^{\circ} 6$ que dirige la Srta. Esquircia he podido apreciar que tanto esta digna Maestra como las itres? (sic) que la sirven, trabajan con buena orientación y espíritu de religiosidad y patriotismo siendo buenos los resultados de la labor realizada" (Santander, 2009, p. 10).

\section{La evidencia de una riqueza personal y social}

Numerosos nombres no incluidos aquí, esperan ser rescatados de un desconocimiento que no nos beneficia. De ahí que gratifique comprobar el interés que despiertan las biografías de mujeres. Porque hacer memoria es, sin duda, un homenaje que debemos a quienes nos han precedido; pero no solo, es también un espejo en el que, detrás de la imagen propia, percibimos una continuidad de rostros, una genealogía de mujeres que nos respaldan, alientan y son un aval de confianza para saber que es posible hacer realidad nuestros deseos.

Los trazos biográficos de las mujeres aquí nombradas nos hablan de su capacidad para desbordar los moldes a que debían adaptarse; para alcanzar con excelencia las metas académicas, para desempeñar una profesión en la que contaban únicamente con referencias 
de hacer masculinas. La inseguridad, si la sintieron, no fue obstáculo paralizante. El estímulo y apoyo familiar, el éxito en forma de calificaciones, y la buena acogida de su actividad profesional, nos hablan de una sociedad que presenció acciones individuales, no bloqueadas por el entorno, de ruptura, de innovación, de evidencia de un yacimiento de riqueza con muchas más posibilidades de las reconocidas y utilizadas hasta el momento.

Eso han querido ser estas páginas, recuerdo de médicas como Antonia Arrobas, María del Mar Terrones, Adelaida Navarro, Herminia Adrados o María Dolores Sarria; de farmacéuticas como Josefa Sarriá y Rosa Herrero; de las maestras Ángeles Gatica y Carmen Abela, la inspectora Teresa Izquierdo Izcue. Estos y otros nombres que aparecen citados merecen un tratamiento biográfico más amplio, que la investigación en historia de las mujeres irá realizando. Lo que aquí aparece ha contado con investigaciones anteriores y ojalá actúe, a la vez, como despertador para seguir ampliando este evocador conocimiento.

\section{Referencias}

Aguilera Sastre, J. (2011). Las fundadoras del Lyceum Club Femenino Español, en Brocar, 35, pp. 65-90.

Almisas Albéndiz, M. (2020). La familia Carvia Bernal y la Facultad de Medicina. Una historia de librepensamiento y masonería en el Cádiz de finales del siglo XIX, Cuadernos de Investigación de Fondos del Archivo UCA, 2, pp. 1-8.

Azcárate Ristori, I. de (2000). Una niña regidora honoraria de la ciudad de Cádiz. Noticia biográfica de D. ${ }^{a} \mathrm{M}^{\mathrm{a}}$ del Rosario Cepeda y Mayo. 1756-1815, Cádiz, Quorum Editores.

Cabrera-Alfonso, J.R., y Herrera Rodríguez, F. (2004). Expedientes de depuración de profesionales sanitarios a través de la documentación existente en el Colegio Oficial de Médicos de Cádiz (1941-1946), en J. Martínez Pérez, Mª I. Porras Gallo, P. Samblás Tilve, M. del Cura Gozález (coords.). La medicina ante el nuevo milenio: una perspectiva histórica, Cuenca, Universidad Castilla-La Mancha, pp. 401-424.

Capel Martínez, R. Ma $(1982,18962)$. El trabajo y la educación de la mujer en España (1900-1930), Madrid, Ministerio de Cultura.

Espigado Tocino, G. (1996). Aprender a leer y escribir en el Cádiz del ochocientos, Cádiz, Servicio de Publicaciones de la Universidad de Cádiz.

Espigado Tocino, G. (2008). La Buena Nueva de la Mujer-Profeta: Identidad y cultura política en las fourieristas M. Josefa Zapata y Margarita Pérez de Celis", Pasado y memoria: Revista de historia contemporánea, 7, pp. 15-33.

Espigado Tocino, G. (2020). Orden legal y orden sexual en los comienzos de la Revolución Septembrina (1868-1870), Memoria y Civilización 23, 187. (167-1909 
Fernández Poza, M. (2003): Frasquita Larrea: Entre la Ilustración y el Romanticismo. Apuntes biográficos de una vida en el umbral de la modernidad, en Pascua, $\mathbf{M}^{\mathrm{a}}$ José de la y Espigado Tocino, Gloria (2003). Frasquita Larrea y Aherán. Europeas y españolas. entre la Ilustración y el Romanticismo (1750-1850), Cádiz, Universidad de Cádiz, pp. 25-53.

Flecha García, C. (1996). Las primeras universitarias en España, Madrid, Narcea.

Flecha García, C. (2010). "Las aspirantes al magisterio secundario en el proyecto de renovación pedagógica de la JAE”, en Sánchez Ron, José M.-García-Velasco, José. La Junta para Ampliación de Estudios e Investigaciones Científicas en su centenario, Madrid, Publs Residencia de Estudiantes, vol. 2, pp. 649-681.

García Garralón M., y Renau López, R. (2020). Pioneras farmacéuticas. Las primeras mujeres del Colegio Oficial de Farmacéuticos de Madrid (1918-1936), Madrid, Colegio Oficial de Farmacéuticos de Madrid.

Hernández Martín, F. (2006). Las Hijas de la Caridad en la profesionalización de la enfermería, Cultura de los Cuidados. Revista de Enfermería y Humanidades, X/20, pp. 39-49.

Heredia Flores, V. M. (2021). Los estudios de Fanny Medina, primera médica colegiada de Andalucía, en la Facultad de Medicina de Cádiz, Cuadernos de Investigación de Fondos del Archivo UCA, n. ${ }^{\circ}$ 3, pp. 14-31.

Herráiz Gascueña, M. G. (1995). Aproximación a la educación especial española del primer tercio del siglo XX, Cuenca, Servicio Publs Universidad de Castilla-La Mancha.

Hoyos Sainz, L. de (1922). Etnografía Española. Cuestionario y Bases para el estudio de los trajes regionales. Actas y memorias de la Sociedad Española de Antropología, Etnografía y Prehistoria, año 1, vol. I, pp. 91-129.

Jiménez Almagro, A. (1984). Estudio biográfico y crítica de Patrocinio de Biedma y La Moneda, Madrid, Centro Estudios Patrimonio Histórico Ambrosio de Morales.

López García, M. P., Morales, A., y Sigler, F. (1995). La mujer en la masonería gaditana. Estudio de las logias, en Ferrer Benimeli José Antonio (coord.). La masonería española entre Europa y América, Zaragoza, Gobierno de Aragón, Vol. 1, pp. 199. 208.

Magallón Portolés, C. (1998). Pioneras españolas en las ciencias. Las mujeres del Instituto Nacional de Física y Química, Madrid, Consejo Superior de Investigaciones Científicas.

Marín Eced, T. (1991). Innovadores de la educación en España, Cuenca, Servicio Publs Universidad Castilla-La Mancha. 
Ortiz García, C- (1987). Luis de hoyos San Sainz y la antropología guía española, Madrid, Consejo Superior de Investigaciones Científicas, pp. 110- 112.

Palacios Bañuelos, L. (1988). Instituto-Escuela. Historia de una renovación educativa, Madrid, Ministerio de Educación y Ciencia.

Perea Carpio, María A. (2004). La educación de la mujer en la narrativa de Patrocinio de Biedma, Boletín Instituto de Estudios Giennenses, 187, pp. 665-792.

Pettenghi, J. A. (2005). La escuela derrotada: depuración y represión del Magisterio en la provincia de Cádiz, Cádiz, Quórum.

Poveda Sanz, M. (2014). Mujeres y segunda enseñanza en Madrid (1931-1939), Tesis Doctoral, Madrid, Universidad Complutense.

Pozo Andrés, Ma del M. del (2013). Justa Freire o la pasión de educar, Barcelona, Octaedro.

Pro Ruiz, J. (2015). Mujeres en un estado ideal: la utopía romántica del fourierismo y la historia de las emociones, Rubrica Contemporánea, 4/7, pp. 27-46.

RE (República Española) (1935). Anales del Museo del pueblo español. Tomo 1, Madrid. Ministerio de Instrucción Pública y Bellas Artes. Portada interior.

Rigada Ramón, María E. de la (1913). Triunfos de la mujer. Gaceta de Instrucción Pública y Bellas Artes, 1210, 13 agosto, p. 519.

Rodríguez Díaz, J. María (2016). Casinos, sindicatos y cofradías: Un siglo de asociaciones en la provincia de Cádiz (1833-1931), Sevilla, Punto Rojo Libros.

Ruiz Guerrero, C. (2011). El pensamiento audaz tiende su vuelo. pioneras del periodismo en Cádiz, Cádiz, Quorum Ediciones.

S/A. More medical stories. Lady Doctors. https://cutt.ly/4lm94q0.

Salaverry Baro, F. (1993). Historia de la Asociación de la Prensa de Cádiz. 1909-1992, Cádiz, Asociación de la Prensa de Cádiz.

Santander Díaz, M. (2009). Inspección de educación de Andalucía: Los Libros de Visitas de Inspección o como la educación se entendió desde la Inspección y de qué manera se reflejaba en los Libros de Visitas, CABÁS, 2, diciembre. https://cutt.ly/tlm9Mpu

Triviño Cabrera, L. (2011). Ellas también pintaban. El sujeto femenino artista en el Cádiz del siglo XIX. Sevilla, Ediciones Alfar. 
Vázquez Ramil, R. (2012). Mujeres y educación en la España contemporánea, Madrid, Akal.

Referencias hemerográficas:

Aguilar Catena, J. (1917). En la Academia Teresiana, La Acción, de 7 de marzo, p. 2.

EL CORRESPONSAL (1912). Reparto de Premios, El Correo de Cádiz, 11 noviembre, pp. $1-2$.

Honahilag (1912). De mi Álbum. Las Sirenas. A la distinguida y joven escritora Srta. María del Mar Terrones, Diana, 31 mayo, p. 4

R. (1954). Sociedad de Educación Internacional, ABC, 31 enero, p. 53.

S/A (1893). Triunfo completo, El Guadalete, Jerez de la Frontera, 17 junio, p. 3.

S/A (1897). El Guadalete, 7 mayo, p. 3; 11 mayo, p. 3; 13 mayo, p. 3; 15 mayo, p. 3; 16 mayo, p. 3; 18 mayo 18 , p. 3; 20 mayo, p. 3; 22 mayo, p. 3; 24 mayo, p. 2; 25 mayo, p. 3.

S/A (1899). De Cádiz, El Guadalete, 14 octubre, p. 2.

S/A (1905). Ayer aparecería en Cádiz, El Guadalete, 11 mayo, p. 2.

S/A (1907). Oposiciones a premios, Gaceta de instrucción pública, 2 octubre, p. 645.

S/A (1908a). Certamen Pedagógico en Alicante, Suplemento a La Escuela Moderna. 28 octubre, pp. 1391-1393.

S/A (1908b). Certamen Pedagógico, Heraldo de Alicante, 25 enero, p. 1.

S/A (1910). Instituto del Cardenal Cisneros, El Siglo futuro, 1 octubre, n. ${ }^{\circ}$ 973, p. 3.

S/A (1911a). Sección de noticias, El Magisterio Español, 27 junio, p. 1026.

S/A (1911b). Notas provinciales, La Información, 14 julio, p. 2.

S/A (1911c). Srta. María del Mar Terrones y Villanueva, Diana, diciembre, portada

S/A (1911d). En Provincias. En Cádiz. La Época, 2 diciembre, p. 2. Artículos de Protesta, La Correspondencia de España, 1 diciembre, p. 5.

S/A (1912a). Conferencias Escolares, El Correo de Cádiz, 14 marzo, p. 1.

S/A (1912b). Acto Académico, El Correo de Cádiz, año, 1 abril, p. 1. 
S/A (1912c). Congreso de periodistas, El Bloque, 5 junio, p. 3.

S/A (1912d). Congreso periodístico, La Palanca, 11 junio, p. 3.

S/A (1912e). Velada en honor de Colombia, España y América, I/3, p. 39.

S/A (1912f). Un banquete. La correspondencia de España, 8 diciembre, p. 3.

S/A (1913). Un banquete y una subasta. La Correspondencia de España, 20 noviembre, p. 2.

S/A (1914a). Alumna interna, El Correo de Cádiz, 27 enero, p. 1.

S/A (1914b). Cuerpo de Enfermeras Gaditanas, El Correo de Cádiz, 5 marzo, p. 1.

S/A (1914c). Academia Teresiana, El Correo de Cádiz, 22 agosto, p. 3.

S/A (1920). En la Inspección Provincial de Primera Enseñanza. El Correo de Cádiz, 27 julio, p. 3.

S/A (1921). El Noticiero Gaditano, 12 septiembre, p. 2. Lábaro Hispano, Algeciras, 7 agosto, p. 3.

S/A (1925a). El Noticiero Gaditano. 6 junio, p. 1. Ibidem, 31 agosto, p. 1.

S/A (1925b). El Noticiero Gaditano, 29 julio, p, 1. Ibidem, 16 julio, p. $3 ; 5$ y 6 agosto, p. 1; 8 septiembre, p. 1; (1926) 24 agosto, p. 1.

S/A (1925c). El Noticiero Gaditano, 2 diciembre, pp. 1-2.

S/A (1927). El Noticiero Gaditano, 10 febrero, p. 1.

S/A (1928). El cursillo de labores, bordados y corte en Cádiz, El liberal, Madrid, 21 marzo, p. 6.

S/A (1930). Los nuevos Inspectores Municipales de Sanidad. El Noticiero Gaditano, 1 noviembre, p. 2.

S/A (1937). Águilas. Diario nacional sindicalista, Cádiz, 6 febrero, II/57, p. 4.

\section{Referencias Normativas:}

GM (Gaceta de Madrid) (1907). Universidad Central. Propuesta formulada por este Rectorado para la provisión por concurso de traslado de las Escuelas y Auxiliarías

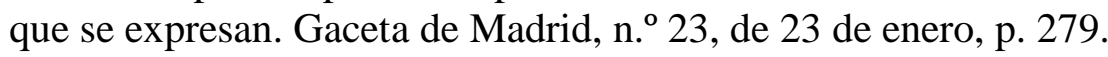


GM (1920). Real orden nombrando Inspectores de Primera enseñanza de las provincias que se mencionan a las señoras que se indican. 24 julio, p. 270.

GM (1933). Dirección general de Primera enseñanza. Rectificaciones nombramientos, provisionales de Maestras, 4 agosto, p. 871.

GM (1934). Decreto de 26 de julio de 1934 creando el museo del Pueblo Español, 28 julio, pp. 965-967.

GM (1935). Orden nombrando a los señores que se mencionan Vocales del Comité de Tutelación Social del Colegio Nacional de Ciegos, 14 mayo, p. 1319.

GR (Gaceta de la República) (1937). Orden separando definitivamente de sus cargos, con pérdida de todos sus derechos, a los Inspectores de Primera Enseñanza que se relacionan, 3 febrero, pp. 625-626.

BOE (1942). Orden por la que se aprueba el concurso de antigüedad o de prelación en la Escalafón del Cuerpo Médico de Asistencia Pública Domiciliaria convocado por la de 29 de octubre de 1941 para proveer en propiedad las plazas comprendidas en la Convocatoria del mismo, adjudicando los nombramientos respectivos a los solicitantes en la siguiente forma, 10 de julio.

BOE (1944) Tribunales de oposición a plazas de Médicos de Asistencia Pública Domiciliaria. Relación de los aspirantes admitidos a la práctica de los ejercicios, 1 de octubre.

BOE (1959) Orden de 10 de noviembre de 1959 por la que se eliminan del Cuerpo y escalafón de Médicos titulares a los facultativos que se relacionan, por haber dejado transcurrir más de diez años en situación de excedencia voluntaria sin reingresar al servicio activo, o por no haber tomado posesión de la plaza de plantilla del Cuerpo que les fue adjudicada en concursos y oposiciones en el período reglamentario, 27 de noviembre.

\section{Referencias Archivísticas:}

AGA (Archivo General de la Administración) (1914). Expediente personal de María del Mar Terrones Villanueva, 32/6363 (005)

AHCM (Archivo Hijas de la Caridad. Provincia de Madrid "San Vicente"). Expediente de Sor Adela Navarro Patiño.

AHN (Archivo Histórico Nacional) (1904). Expediente escolar de Rosa herrera Montenegro, Universidades, 2495, Exp.27. Expediente académico de..., Universidades, 5712, Exp.32. 
AGA (1896). Expediente Académico de Antonia Monreal Andrés, Educación y Ciencia. Caja 16255.

AUCA (Archivo Histórico Universidad de Cádiz) (1889). Expediente de Antonia Monreal Andrés, 1889-1895, C-159 (1)-6 FM

AUCA (1910). Expediente personal de María del Mar Terrones Villanueva, 1910-1915, C249 (2)-17 FM

AUCA (1924). Expediente personal de Adelaida Navarro Patiño, 1924-1940, C-219-2 FM

AUCA (1939). Expediente personal de María Dolores Sarriá Guerrero, 1939-1947, C-342 (3)-68FM

AUG (Archivo Universitario Universidad de Granada) (1917): Expediente Académico de Josefa de Sarriá Guerrero, 1917-1921, ES AUG I 143 PRINCIPAL. Caja 00145 / 027

AGUCM (Archivo General de la Universidad Complutense de Madrid). Expediente personal de María del Mar Terrones Villanueva. Facultad de Medicina, ES, AGUCM, M-677, 33. 